\title{
Esforços da pesquisa brasileira sobre mineração e impactos ambientais: uma visão geral de cinco décadas (1967-2017)
}

Objetivou nesse trabalho analisar as tendências e lacunas dos estudos sobre mineração e impactos ambientais no Brasil nas últimas cinco décadas (1967 - 2017 ) através de análise cienciométrica. A pesquisa ocorreu através de busca de trabalhos com combinações de palavras, nas bases de dados ISI Web of Knowledge (Thomson Reuters), Scopus Database (Elsevier) e Scielo (Scientific Electronic Library Online). Foram tabulados 550 trabalhos. Existe uma correlação forte positiva entre o número de trabalhos e ano de publicação $(r=0,827 ; p<0,001)$, há um crescimento significativo de publicações ao longo dos anos. A maior parte dos estudos foram realizados em ambiente terrestre (solo) (32,99\%). As principais abordagens dos estudos são análise físico/química (34,91\%). Foi encontrado uma alta diversidade de periódicos que publicam trabalhos sobre mineração no Brasil $\left(H^{\prime}=4.653\right)$, sendo Revista Escola de Minas $(6,36 \%)$ o periódico com maior número de publicações. O estado com maior número de publicações foi Minas Gerais (16,43\%). Os minérios mais relacionados aos estudos foram ouro (26,19\%). Alteração da qualidade do solo $(26,35 \%)$ têm sido o impacto mais estudado em trabalhos sobre mineração. A área de atuação dos pesquisadores com maior frequência de ocorrência foi Ciências Biológicas (43,27\%). Avaliar as métricas de produção científica da área de mineração no Brasil, permitiu identificar as lacunas, fator que contribui para que possíveis novos pesquisadores possam vir a realizar estudos que contemplem essa temática, salientando a necessidade de se descentralizar as pesquisas dos maiores centros urbanos do país e expandi-las as demais localidades.

Palavras-chave: Mineração; Tendências de Publicação; Lacunas do Conhecimento.

\section{Brazilian research efforts on mining and environmental impacts: an overview of the five decades (1967-2017)}

\begin{abstract}
The objective of this work was to analyze the trends and gaps of the mining and environment impacts studies in Brazil in the last five decades (1967 - 2017) through a scientometric analysis. The research was carried out by searching for works with combinations of words in the ISI Web of Knowledge (Thomson Reuters), Scopus Database (Elsevier) and Scielo (Scientific Electronic Library Online) databases. 550 papers were tabulated. There is a strong positive correlation between the number of papers and year of publication $(r=0.827 ; p<0.001)$, there has been a significant growth of publications over the years. Most of the studies were performed in terrestrial environment (soil) (32.99\%). The main approaches of the studies are physical/chemical analysis (34.91\%). It was found a high diversity of periodicals that publish works on mining in Brazil ( $\left.\mathrm{H}^{\prime}=4.653\right)$, being Revista Escola de Minas $(6.36 \%)$ the periodical with the greatest number of publications. The state with the highest number of publications was Minas Gerais (16.43\%). The minerals most related to the studies were gold (26.19\%). Changes in soil quality (26.35\%) have been the most studied impact on mining work. The area of action of the researchers with the highest frequency of occurrence was Biological Sciences (43.27\%) To evaluate the metrics of scientific production in the mining area in Brazil, allowed to identify the gaps, a factor that contributes so that possible new researchers can carry out studies that contemplate this subject, emphasizing the need to decentralize the surveys of the largest urban centers of the country and expand them to other locations.
\end{abstract}

Keywords: Mining; Publishing Trends; Knowledge Gaps

Topic: Desenvolvimento, Sustentabilidade e Meio Ambiente

Reviewed anonymously in the process of blind peer.
Received: 05/02/2020

Approved: 09/03/2020
Sabrina Santos da Costa (iD

Universidade Federal do Oeste do Pará, Brasi

http://lattes.cnpq.br/5987680798801956

http://orcid.org/0000-0002-0661-8173

sabrina.costt@gmail.com

Leônidas Luiz Volcato Descovi Filho (iD)

Universidade Federal do Oeste do Pará, Brasil

http://lattes.cnpq.br/5987680798801956

http://orcid.org/0000-0002-0661-8173

leonprs@gmail.com

José Max Barbosa de Oliveira Junior (iD

Universidade Federal do Oeste do Pará, Brasil

http://lattes.cnpq.br/1353014365045558

http://orcid.org/0000-0002-0689-205X

maxbio@hotmail.com

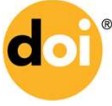

DOI: $10.6008 / C B P C 2179-6858.2020 .002 .0029$
Referencing this:

COSTA, S. S.; DESCOVI FILHO, L. L. V.; OLIVEIRA JUNIOR, J. M. B.. Esforços da pesquisa brasileira sobre mineração e impactos ambientais: uma visão geral de cinco décadas (1967-2017). Revista Ibero Americana de Ciências Ambientais, v.11, n.2, p.296-313, 2020. DOI: http://doi.org/10.6008/CBPC2179-6858.2020.002.0029 


\section{INTRODUÇÃO}

A atividade de mineração representa na história um grande destaque devido a manutenção e melhoria da qualidade de vida humana, da produção e do consumo ser proporcionada através da exploração dos bens minerais (WEBER, 2014). No segundo semestre do ano de 2017 o Brasil apresentou índices de aumento de $4,9 \%$ de produção de minérios, no quais o estado brasileiro que mais exportou foi o estado de Minas Gerais, a substância mineral mais produzida e exportada foi o ferro, representando $63,8 \%$ das exportações (BRASIL, 2017).

O Brasil possui grande potencial de se estabelecer como um dos maiores produtores minerais a nível mundial, para que ocorra a exploração com maior benefício econômico dos depósitos minerais, é imprescindível dispor de desenvolvimento de tecnologias específicas que possibilitem o acúmulo dos minerais de minérios, e assim como no processo de extração os seus elementos químicos sejam retirados através de técnicas sustentáveis. 0 país também possui potencialidade para ser uma liderança global na criação e exportação de tecnologia mineral (LINS, 2016).

Contudo, as atividades mineradoras podem ocasionar danos socioambientais de grandes proporções muitas vezes irrecuperáveis, com impactos aos componentes do meio ambiente como fauna, grandes áreas de vegetação, entre outros, enquanto ao social os impactos estão relacionados ao aumento de violência urbana, precarização da força de trabalho e transformação socioespacial construída historicamente (PASSOS et al., 2017).

Os estudos da cienciometria estão propostos a estimar a produção científica, através dos insumos e resultados conhecidos como indicadores cienciométricos, também com a utilização de técnicas e análises estatísticas que são grandemente discutidas e validadas (RAZERA, 2016). No Brasil a cienciometria tem crescido significativamente, esse aumento de trabalhos cienciométricos é indicado por alguns elementos, dentre eles: (i) grupos de pesquisas cienciométricas se consolidando; (ii) volume crescente de produção; (iii) existência de um evento especializado no assunto, que é realizado a cada dois anos; (iv) previsão de criação de um periódico nacional temático (ALVARADO, 2014).

Considerando a possibilidade de melhor compreender processos e impactos ocorridos nos estados brasileiros e o desenvolvimento de tecnologias no setor mineral, este trabalho teve como objetivo analisar as tendências e lacunas dos estudos sobre mineração e impactos ambientais no Brasil nas últimas cinco décadas (1967-2017) através de uma análise cienciométrica.

\section{REFERENCIAL TEÓRICO}

\section{Panorama da mineração no Brasil}

A produção de exploração mineral é de grande importância para o desenvolvimento das atividades modernas, desde os setores de infraestrutura básica como no desenvolvimento de novas tecnologias. Quando se fala em mineração a nível mundial o Brasil se caracteriza como um dos principais produtores e exportadores, tem uma produção de 72 substâncias minerais, no qual estão classificadas em 23 metálicas, 
45 não metálicas e 4 energéticas. O líder da produção mineral é o ferro com $60 \%$ do valor total da produção, em seguida o ouro como o valor de 5\% (ARAUJO et al., 2014).

De acordo com o Departamento Nacional de Produção Mineral do Brasil - DNPM (2017) o país no ano de 2015 teve índices significativos em relação ao aumento da exploração mineral, principalmente da classe dos metálicos, no quais foram destacados os metais: alumínio, cobre, estanho, ferro, manganês, nióbio, níquel e ouro. O valor da produção comercializada dessas oito substâncias totalizou 67,5 bilhões de reais, com destaque para a participação do ferro, cuja produção é concentrada, principalmente, nos estados de Minas Gerais e Pará.

De acordo com Brasil (2016) as exportações brasileiras de minérios foram destinadas aos países: China, Japão, Estados Unidos, Canadá e países baixos. As minas com maiores concentrações de minérios estão localizadas nas regiões sudeste e norte. As principais empresas produtoras de alumínio (bauxita) são Mineração Rio do Norte, Mineração Paragamominas e Alcoa World Alumina. As empresas que se destacaram na produção de ferro foram a Vale S.A, Companhia Siderúrgica Nacional, Samarco Mineração S.A. As empresas com maiores produções de ouro: Kinross Brasil Mineração S.A, Anglogold Ashanti Córrego Do Sítio Mineração S.A e Salobo Metais S.A.

Em se tratando de investimento em pesquisa e desenvolvimento na mineração, o Brasil apresenta déficit no investimento no setor mineral, quando comparado com outros setores de exploração de recursos naturais. O investimento inadequado reflete no baixo número de pesquisadores e técnicos de instituições de pesquisa e desenvolvimento exclusivos para estudar cada particularidade do setor da mineração, também reflete em uma lacuna nessa temática, no qual a reforma se faz necessária para que o país não fique detido aos avanços tecnológicos desenvolvidos em outros países (LINS, 2016).

\section{Impactos socioambientais da mineração}

Os impactos e alterações ambientais se tornam presentes na mudança de temperatura, clima e no meio ambiente como um todo, dentre os impactos causados pelas atividades de extração de minérios, estão mudanças na paisagem, impactos na cobertura vegetal, na biodiversidade do solo e, consequentemente, nos ciclos biogeoquímicos dos elementos uma vez que o processo de abertura de poços de mineração envolve a remoção do solo sobre o minério (que é estéril) e o depósito em outros locais, formando pilhas (XING et al., 2015).

Para Mota et al. (2017), a mineração é um setor que tem compromissos com a sustentabilidade, partindo desse princípio o autor analisa o encontro entre racionalidade econômica e ambiental por parte da indústria mineral, que hoje por responsabilidade social e também exigência do mercado internacional estão cada vez mais investindo em projetos socioambientais no combate aos impactos causados por atividade de exploração, principalmente na região da Amazônia.

Contudo, com a abertura comercial e exploratória da mineração no país, moradores de áreas próximas as mineradoras têm sofrido conflitos socioambientais devido ao aumento da população nas cidades, precarização das forças de trabalho e aumento nos casos de violência urbana, além de doenças 
ocasionadas por contaminação de metais pesados utilizados para extrair minérios como o ouro. Estudos fornecem informações relevantes para instrumentalizar ações de planejamento de uso e ocupação do solo e de mitigação de efeitos de danos ambientais e conflitos socioambientais associados (PASSOS et al., 2017).

$\mathrm{Na}$ análise de Mello (2015), o autor descreve sobre o carreamento de sedimentos para os corpos hídricos do entorno de uma mineração ao céu aberto que pode alterar o regime natural de descarga sólida ao introduzir grandes volumes de sedimentos ao meio ambiente. Com efeito, à jusante do empreendimento pode ocorrer o aumento da turbidez provocada por sedimentos finos em suspensão, assim como, a poluição causada por substâncias lixiviadas, carreadas ou contidas nos efluentes das áreas de mineração, afetando a fauna e flora aquática.

Vieira et al. (2015), compreendem que a mineração é considerada um dos setores básicos da economia no Brasil. A atividade minerária fornece matéria-prima para a indústria, sendo que vários produtos, desde os mais simples aos mais complexos, têm origem mineral, fica evidente a relação de dependência da sociedade moderna e o setor de mineração, que gera renda para muitos. As atividades de extração são de grande importância para o desenvolvimento nacional, mas igualmente responsáveis por impactos ambientais negativos, alguns inclusive irreversíveis que precisam de técnicas para recuperação das áreas afetadas.

\section{Cienciometria}

O processo de avaliação da ciência não é um método de pesquisa novo no meio acadêmico no qual dentro desse procedimento de avaliação as abordagens de pesquisas que mais se destacam é a pesquisa bibliográfica ou revisão de literatura e cienciometria que desempenham um papel fundamental na comunicabilidade de informações entre pesquisadores (CABRAL-NETTO et al., 2015).

A análise cienciométrica é multidimensional e requer a análise combinada de diferentes indicadores, possui temas de interesses que estão relacionados ao crescimento e desenvolvimento das áreas e campos científicos. Nesse sentido, a cienciometria pode ser realizada com os objetivos de identificar as tendências e o crescimento do conhecimento em uma área; determinar o desempenho dos sistemas de recuperação da informação; avaliar os aspectos estatísticos da linguagem, das palavras e das frases; medir o crescimento de determinadas áreas e o surgimento de novos temas (VANTI, 2002).

A cienciometria contribui nas diferentes áreas do conhecimento da ciência, ela permite preencher lacunas do campo científico de determinada área de estudo, e observar quais questões estão inseridas numa dimensão métrica ainda fora do alcance ou do contexto das pesquisas avaliativas de preponderância qualitativa realizadas (RAZERA, 2016).

De acordo com Spinak (1996) o conceito de cienciometria foi progredindo ao longo dos anos. As primeiras definições limitavam-se à medição da informação. Atualmente, o conceito faz menção às leis, propriedades e características diversas que subjazem uma dimensão interdisciplinar da comunicação científica.

A cienciometria é um segmento da sociologia da ciência, sendo aplicada no desenvolvimento de políticas científicas. Envolve estudos quantitativos das atividades científicas, incluindo a publicação, ela não 
pode substituir um método analítico sobre determinado assunto, mas tem a capacidade de provocar maior visibilidade dos dados da pesquisa. Tal recurso é importante para identificar quais áreas precisam de maior preocupação (LAURINDO et al., 2010).

O avanço da ciência da informação e das ciências em geral se dá pela constante elaboração de novas pesquisas e pela concretização e divulgação de seus resultados em diferentes tipos de suportes. Nesse contexto, a cienciometria procura estudar aspectos quantitativos da ciência e da produção científica, quer como uma disciplina ou como uma atividade econômica. Assim, as técnicas cienciométricas são importantes para identificar as tendências e o desenvolvimento do conhecimento, bem como sintetizar o conhecimento com o intuito de identificar lacunas para futuros trabalhos (AKERMAN, 2013).

De acordo com Macias-Chapula (1998), a análise cienciométrica é o estudo dos aspectos quantitativos da produção, disseminação e uso da informação desenvolvendo padrões e modelos para quantificar esses processos usando seus resultados para a elaboração de previsões apoiando a tomada de novas resoluções.

\section{MATERIAIS E MÉTODOS}

\section{Tipo de estudo}

O método de pesquisa caracteriza-se como cienciométrico, é um estudo quantitativo, onde os artigos selecionados foram agrupados em categorias para analisar os principais indicadores cienciométricos dos temas abordados na literatura científica, para responder os objetivos da pesquisa. Cada literatura foi categorizada utilizando os principais indicadores cienciométricos e de importância no cenário nacional sobre o tema mineração (MACIAS-CHAPULA, 1998).

\section{Levantamento de dados}

A pesquisa foi realizada através de uma busca quantitativa de trabalhos científicos publicados nos últimos 50 anos (1967 a 2017) sobre mineração no Brasil. A pesquisa foi feita em três bases de dados internacionalmente reconhecidas: ISI Web of Knowledge (Thomson Reuters), Scopus Database (Elsevier) e Scielo (Scientific Electronic Library Online), as bases de dados foram acessadas através do Portal de periódicos da Coordenação de Aperfeiçoamento de Pessoal de Nível Superior-CAPES.

A escolha destas bases de dados se deu pelo grande impacto que elas possuem para ciência. A ISI Web of Knowledge (Thomson Reuters) é uma base de dados que há anos têm sido a ferramenta disponível de indexação de literatura científica e a mais reconhecida internacionalmente, fornece importantes dados sobre as mais diferentes áreas de pesquisas em ciências e tecnologia (PEREIRA et al., 2018). A Scopus Database (Elsevier) têm apresentado um aumento significativo no número de artigos indexados nas últimas quatro décadas (ALVES et al., 2014). A Scielo foi adicionada por abranger trabalhos locais e/ou regionais, publicando mais de 380 revistas científicas essa base de dados inclui artigos de temáticas variadas (PUCCINI et al., 2015). Todas essas bases de dados têm sido utilizadas com frequência em estudos cienciométricos 
(PEREIRA et al., 2018; NICACIO et al., 2015).

Padronizou-se um conjunto de palavras nos idiomas português, inglês e espanhol (mineração e brasil; mineradora e brasil; mining e brazil; mining company e brazil; mineria e brasil). Foram utilizadas essas combinações em cada base de dados para realizar a busca. Todos os artigos que tinham esses termos nos títulos, resumos e palavras-chave foram tabulados no software Microsoft Office Excel 2016 para posterior realização de análises quantitativas.

Nos trabalhos, selecionou-se apenas aqueles que respondiam os objetivos do trabalho. Foram extraídas as seguintes informações das produções científicas: (i) ano de publicação, (ii) tipos de ambientes, (iii) abordagens nos estudos, (iv) periódicos, (v) estados, região e bioma onde os estudos foram realizados, (vi) tipo de minério, (vii) tipos de impactos socioambientais relacionados ao trabalho, (viii) e área de atuação do primeiro autor.

\section{Refinamento dos dados}

Para extrair as informações de trabalhos que estavam de acordo com os objetivos da pesquisa, foram aplicadas metodologias de refinamento de informações nas bases de dados selecionadas. No qual o refinamento inicial foi feito a seleção das bases de dados, seguindo para o refinamento posterior, no qual escolheu-se os tipos de produção científica de interesse, selecionando assim apenas os documentos: artigos, revisões e notas, publicados entre 1967 a 2017. Através dos refinamentos anteriores obteve-se resultados globais, nos quais se selecionou os trabalhos publicados e realizados apenas no Brasil, obtendo assim resultados de publicações a nível nacional.

Não foram inclusos no banco de dados desta revisão resumo de congressos e conferências, livros e capítulos de livros, e os trabalhos de periódicos não indexados nas bases de dados acima. Os trabalhos selecionados foram agrupados nas categorias em que foram extraídas as informações e de acordo com a ordem que as bases foram acessadas com intuito de serem analisados estatisticamente e verificar a repetição de trabalhos nas bases de dados, os quais não foram contabilizados (Figura 1).

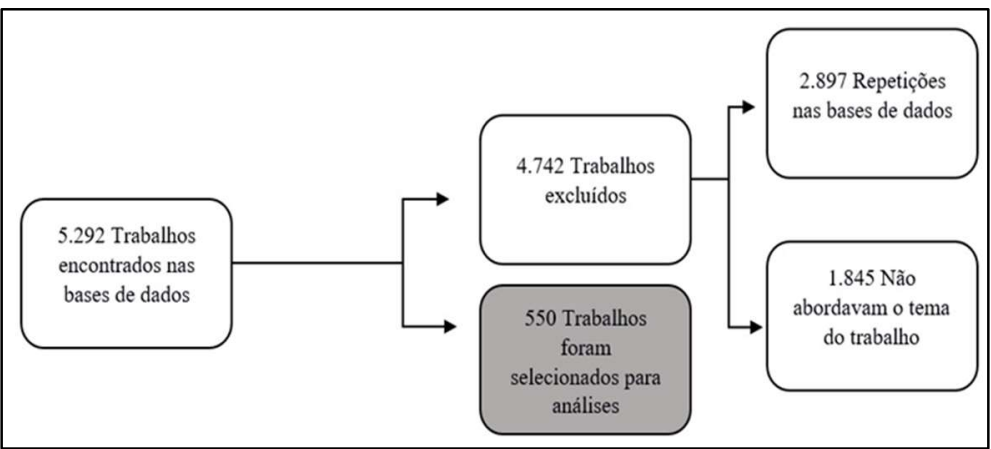

Figura 1: Fluxograma de compilação de dados sobre trabalhos com mineração no Brasil nas últimas cinco décadas (1967 - 2017).

\section{Análise dos dados}

A partir dos dados levantados, os trabalhos foram agrupados por ano de publicação, a fim de facilitar a quantificação, e organizados de maneira sistemática em categorias relacionadas aos temas abordados em 
cada trabalho: tipo de abordagens, tipos de ambientes, periódicos, estados, regiões, biomas, minérios, impactos socioambientais e área de atuação do primeiro autor. Vale ressaltar que para classificar as áreas de atuação dos autores foi agrupado as áreas de atuação de acordo com a classificação da Capes.

Os dados obtidos foram analisados através de análises de frequência simples com intuito de verificar a ocorrência de trabalhos, os quais os resultados foram expressos em gráficos dos principais tipos de ambientes estudados, abordagens de estudos, periódicos de publicações, área de atuação dos autores, tabela dos minérios estudados, mapas dos estados, regiões e biomas.

Para avaliar se existe uma aproximação entre as variáveis trabalhos e ano de publicação foi realizado uma correlação de Pearson (FIGUEIREDO-FILHO et al., 2009). Para testar as diferenças entre trabalhos desenvolvidos em ambientes terrestre e aquático utilizou-se o teste T de student (BARBOSA et al., 2010). Para avaliar se há diferença significativa entre os grupos de impactos socioambientais foi realizada uma Análise de Variância (ANOVA) (MARÔCO, 2018). Para medir a diversidade de periódicos que publicaram artigos sobre mineração nas últimas cinco décadas foi realizado o índice de Shannon-Wiener $\left(H^{\prime}\right)$. Uma Análise de Coordenadas Principais (PCoA) foi utilizada para resumir e visualizar a relação do tipo de impactos e ambientes estudados (aquático e terrestre). A medida de distância utilizada nessa análise foi o índice de Bray-Curtis a partir de uma matriz logaritmizada (log+1) (LEGENDRE et al., 1998). Todas as análises foram realizadas pelas rotinas do programa $\mathrm{R}$ ( $\mathrm{R}$ Development Core Team, 2014) utilizando os pacotes vegan, MASS, MGCV, ADE4, permute e lattice.

\section{RESULTADOS E DISCUSSÃO}

Foi encontrado um total de 5.292 trabalhos devido a abrangência que as combinações utilizadas possuem, no qual foram excluídos 4.742, pelos motivos de: 2.897 trabalhos estarem repetidos nas bases de dados e por 1.845 não abordarem a temática do trabalho. Portanto obteve-se 550 trabalhos relacionados a mineração dos anos de 1987 a 2017 que correspondiam aos objetivos do presente estudo.

\section{Tendências temporais das publicações}

As últimas cinco décadas apresentaram variações nos anos de publicações científicas, observa-se apenas alguns picos de publicações, variando de uma a 47 nesse período. Pode-se observar que a publicação mais antiga encontrada foi no ano de $1987(n=1)$, mantendo-se instável até 1993, com um pequeno pico no ano de 1995 ( $n=13 ; 2,36 \%)$, com variações ao longo dos anos e apresentando picos maiores nos anos de 2015 ( $n=51 ; 9,26 \%)$ e 2017 ( $n=89 ; 16,15 \%)$. Existe uma correlação forte positiva entre o número de trabalhos e ano de publicação $(r=0,827 ; p<0,001)$, há um crescimento significativo de publicações ao longo dos anos (Figura 2).

No ano de 1995 houve um pequeno aumento na quantidade de trabalhos, logo isso pode ser associado ao fato de que nesse período ocorreu um estímulo financeiro na ciência, na década de 90 houve uma pequena ampliação da oferta de bolsas de fomento, incentivos e sistemas de avaliação de méritos nas universidades e da melhoria da cobertura dos periódicos que pode ter colaborado para as pesquisas nesse 
período (MUGNAINI et al., 2004).

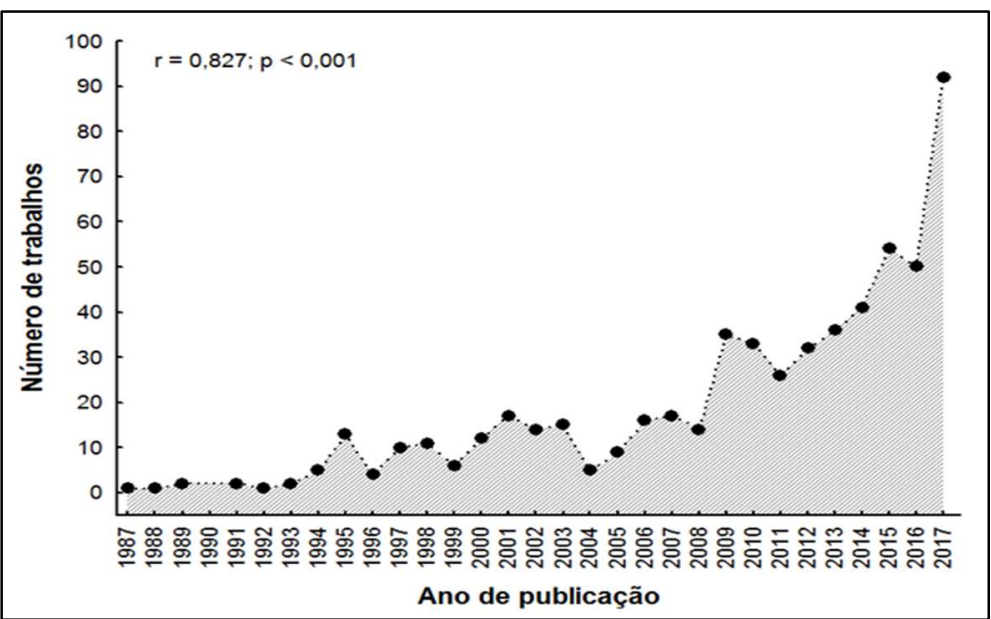

Figura 2: Correlação entre número e ano de publicação dos trabalhos publicados entre os anos de 1967 a 2017.

Além de que nos anos 2003 a 2014, houve um incremento de 18 novas universidades federais, democratização no acesso a pós- graduação e pesquisa com bolsas de auxílio, conforme apresenta os resultados do estudo realizado por Almeida et al. (2013), os autores também informam que a produção científica do Brasil tem aumentado substancialmente, sendo que nos últimos 30 anos houve um crescimento médio de 10,7\% ao ano, cerca de cinco vezes maior que a média mundial, ocupando o 13 no ranking internacional, fato este que pode estar relacionado ao aumento de publicações na temática de mineração.

A maior tendência das pesquisas na área da mineração foi mais expressiva nos anos de 2015 e 2017, este crescente interesse pode ser justificado pelo motivo de no ano de 2015 o país ter apresentado o caso de acidente com rejeitos na área de mineração, o rompimento de uma barragem contendo resíduos provenientes da extração de minério de ferro atingindo toda a bacia hidrográfica do Rio Doce e as cidades pertencentes a essa bacia (ROCHA et al., 2016).

\section{Tipos de ambientes de realização dos estudos}

Não foi encontrada diferença significativa entre o número de trabalhos desenvolvidos em ambientes aquáticos (agrupamento dos ambientes: rio, lago, água subterrânea e lagoa) e terrestres (agrupamento dos ambientes: solo, população humana, vegetação, fauna e ar) ( $T=0,998 ; G . L .=106 ; p=0,320)$. Porém quando subdivididos esses ambientes, nota-se que a maior parte dos estudos foram realizados em ambiente terrestre (solo) ( $n=190 ; 32,99 \%$ ), seguido por aquático (rio) (163; 28,30\%) e terrestre (população humana) ( $n=114$; 11,46\%) (Figura 3).

O maior quantitativo de pesquisas direcionadas a estudar características do ambiente terrestre (solo) podem estar relacionadas a este ambiente sofrer maiores impactos diretos das atividades de mineração a céu aberto nos processos de extração de minérios, uma vez que promove a alteração das propriedades físicas, químicas e biológicas do meio onde está inserida, devido a supressão da vegetação e a remoção dos horizontes superficiais do solo (TEIXEIRA et al., 2017). Os trabalhos também podem estar associados à procura de novas técnicas de recuperação do solo, para restaurar o sistema degradado devido a demanda 
das mineradoras para se adequarem as legislações vigentes (REIS et al., 2014).

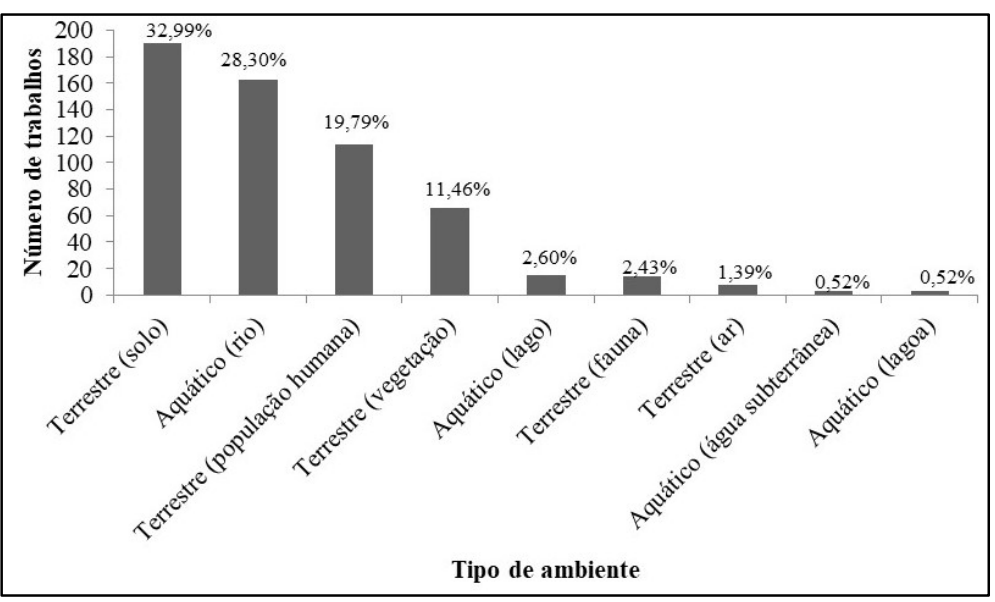

Figura 3: Ambientes relacionados a área de mineração no Brasil mais estudados nas últimas cinco décadas (1967 2017).

Os estudos desenvolvidos em ambiente terrestre (solo) têm se concentrado em avaliar a recuperação do ambiente solo degradado através de técnicas vegetativas como a nucleação, fitorremediação, biorremediação e restauração ecológica, o qual promoverá a rugosidades do solo para que possa desencadear espacial e temporalmente, padrões e propriedades ambientais emergentes devido a hipersensibilidade as condições iniciais do terreno degradado (AUMOND et al., 2014).

\section{Abordagens dos trabalhos}

Os estudos em sua maior parte utilizaram abordagens físico/química ( $n=192 ; 34,91 \%)$, seguido de saúde ( $n=91 ; 16,55 \%)$ e socioambiental e política ( $n=89 ; 16,18 \%)$ (Figura 4$)$.

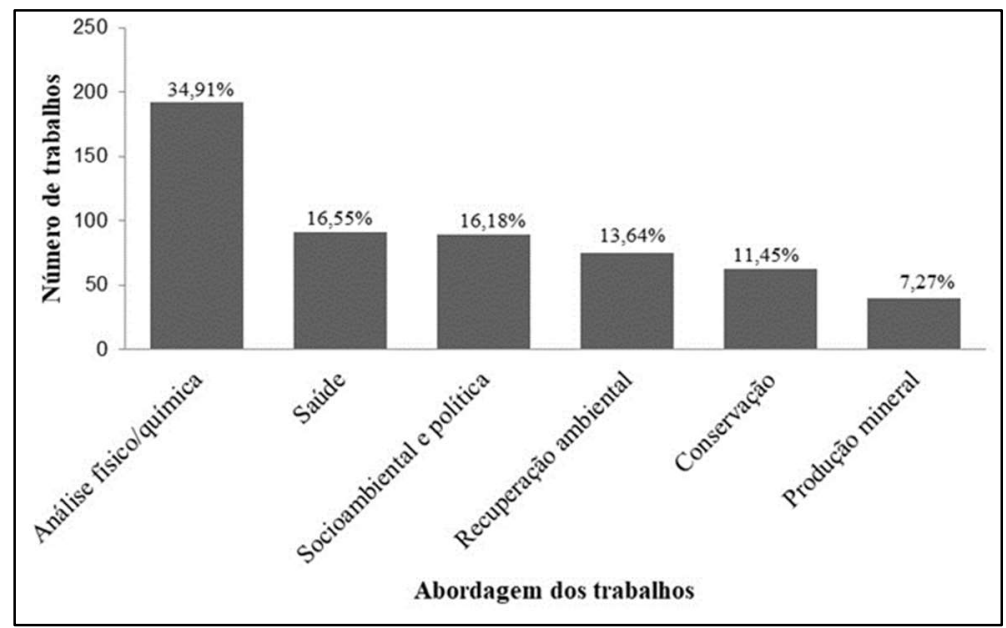

Figura 4: Principais abordagens utilizadas nos trabalhos publicados sobre mineração no Brasil nas últimas cinco décadas (1967 a 2017).

Os trabalhos que utilizaram a abordagem físico/química estavam voltados a determinar o nível de contaminação decorrentes dos processos minerários em ambiente terrestre (solo), principalmente por metais pesados como chumbo, cobre, mercúrio e outros, este fato pode ter contribuído para o maior número de abordagens nos trabalhos terem sido feita através de análises físico/químicas.

Para Santos et al. (2015) a determinação dos teores disponíveis de chumbo no solo é uma ferramenta 
importante para monitorar os riscos de contaminação desses metais no ambiente, pois quando absorvidos pelas plantas os metais podem ser transportados para a parte aérea e serem bioacumulados na cadeia trófica. Além de que estas pesquisas possibilitam auxiliar os planos de recuperação das áreas degradadas necessários para as mineradoras.

Os trabalhos também estavam direcionados a avaliar a eficácia das etapas de recuperação de solos, pois é necessário verificar os solos que passaram pelo processo de degradação decorrente da mineração que foram recuperados e comparar com aqueles que ainda não passaram pela recuperação da área, através de técnicas físico/químicas, para poder avaliar se a área está sendo recuperada de forma eficiente (BITENCOURT et al., 2015).

\section{Principais periódicos que publicaram os trabalhos}

Foi encontrada uma alta diversidade de revistas que publicam trabalhos sobre mineração no Brasil $\left(H^{\prime}=4.653\right)$. Os trabalhos científicos foram encontrados em 207 periódicos diferentes, em relação aos que mais apresentaram frequência de ocorrência foram: Revista Escola de Minas ( $n=35 ; 6,36 \%$ ), seguida pela Science of the Total Environment ( $n=32 ; 5,82 \%$ ) e Revista Brasileira de Ciência do solo ( $n=26 ; 4,73 \%$ ) (Figura $5)$.

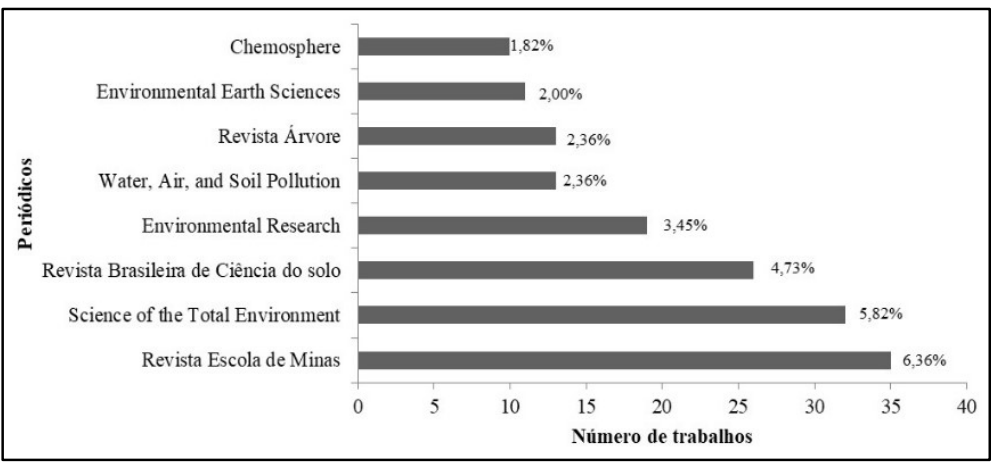

Figura 5: Periódicos com maior número de publicações sobre mineração no Brasil nas últimas cinco décadas (1967 a 2017).

O periódico com maior destaque na quantidade de publicações foi a Revista Escola de Minas ou Revista Internacional de Engenharia, que atua no mercado há 82 anos, é uma das primeiras revistas técnicas (mineração e metalurgia) da América do Sul, especializou-se na publicação de artigos sobre as áreas de Engenharia Civil, Geológica, Metalúrgica e de Materiais, Mecânica e Energia, e Mineração, é indexada na base de dados Scielo.

Em segundo lugar a Science of the Total Environment, é uma revista internacional destinada a publicação de pesquisas originais sobre o meio ambiente, que inclui a atmosfera, a hidrosfera, a biosfera, a litosfera e a antroposfera, surgiu no ano de 1972 e é indexada na base de dados ISI Web of Knowledge (Thomson Reuters), priorizando estudos laboratoriais. A Revista Brasileira de Ciências do Solo também teve destaque na publicação de trabalhos, possui reconhecimento em nível internacional na área de Ciências do solo, publica artigos de pesquisadores brasileiros e internacionais.

Cada área da ciência possui suas preferências quanto a comunicação científica escolhida para expor 
os resultados de suas pesquisas. Em cada um dos grandes campos do conhecimento, os pesquisadores divergem quanto ao canal mais apropriado à divulgação científica e busca de informações. Os periódicos compõem um dos canais mais utilizados para a comunicação de resultados de pesquisa, através deles, o pesquisador publica seus artigos para expor ideias, garantir a propriedade científica e se submeter à avaliação dos pares (CINTRA, 2018).

A produção científica é um instrumento que transmite à comunidade técnico-científica o conhecimento de novas descobertas, e o desenvolvimento de novos materiais, técnicas e métodos de análise nas diversas áreas da ciência. Por meio de uma publicação científica que a sociedade toma conhecimento dos resultados de um trabalho de pesquisa e o que este representa para a coletividade (BROFMAN, 2012).

\section{Tendências espaciais dos trabalhos}

Dentre os 24 estados e o distrito federal estudados nos trabalhos, o estado de Minas Gerais foi o que mais se destacou, ( $n=163 ; 16,43 \%)$, seguido do Pará $(n=112 ; 11,29 \%)$ e Santa Catarina $(n=76 ; 7,66 \%)$. Os estados de Alagoas e Sergipe não apresentaram trabalhos publicados sobre mineração (Figura 6A). Observase que o Sudeste foi a região brasileira mais estudada $(33,6 \% ; n=207)$, seguida do Norte $(25 \% ; n=154)$ e Sul $(22,89 \% ; n=141)$, a região com menos estudos foi o Centro-Oeste $(8,44 \% ; n=52)$ (Figura 6B). Entre os biomas pesquisados, a Mata Atlântica foi estudada por $(29,26 \%$; $n=187)$ trabalhos, seguida pelo Cerrado $(24,41 \%)$ e Amazônia (25,2\%; n= 161), o bioma menos estudado foi o Pantanal (4,38\%; 28) (Figura 6C).

O estado de Minas Gerais foi o mais estudado, possui áreas com alta concentração de ferro (Fe) é o maior produtor do país responsável por aproximadamente 70\% do minério extraído, contribuindo para que o Brasil ocupasse a terceira posição mundial de produção de ferro (BRASIL, 2016). Com áreas de grandes destaques de produção como a parte central de Minas Gerais que é conhecida como Quadrilátero Ferrífero, de grande importância histórico-cultural e econômica devido à extração de minério de ferro, o que pode estar correlacionado a grande quantidade de trabalhos desenvolvidos neste estado (OMACHI, 2015).

Fato que pode ser considerado como justificativa para a região Sudeste ter um índice elevado de publicações é que nesta região está localizado os maiores centros urbanos, no quais encontram-se as maiores e renomadas universidades do país, com grande aporte de investimentos para pesquisa (CHIARINI et al., 2014).

Um estudo realizado por Sidon et al. (2016) corroboram com resultados obtidos, quando afirmam que os estados localizados na região Sudeste são um dos principais produtores de produção da científica brasileira, com cerca de 31\% do total nacional no triênio 2007-2009, o que corresponde a quase 891.779 produções contabilizadas nesse período. Estudos cienciométricos em outras temáticas também tem demostrado que as regiões sudeste e sul apresentam maior quantidade de trabalhos desenvolvidos (FANTINCRUZ et al., 2015; DORNELES, 2016).

Os biomas mais estudados foram a Mata Atlântica e Cerrado, isso pode estar relacionado a localização dos estados e regiões que foram mais estudados na temática dos últimos 50 anos como apresenta este estudo, assim como outras pesquisas cienciométricas de temáticas diferentes também tem apontado o 
bioma Mata Atlântica e Cerrado como os mais estudados e esta relação estaria interligada a estes biomas abranger as regiões Sul e Sudeste (CAMPOS et al., 2014; BATISTA et al., 2016).

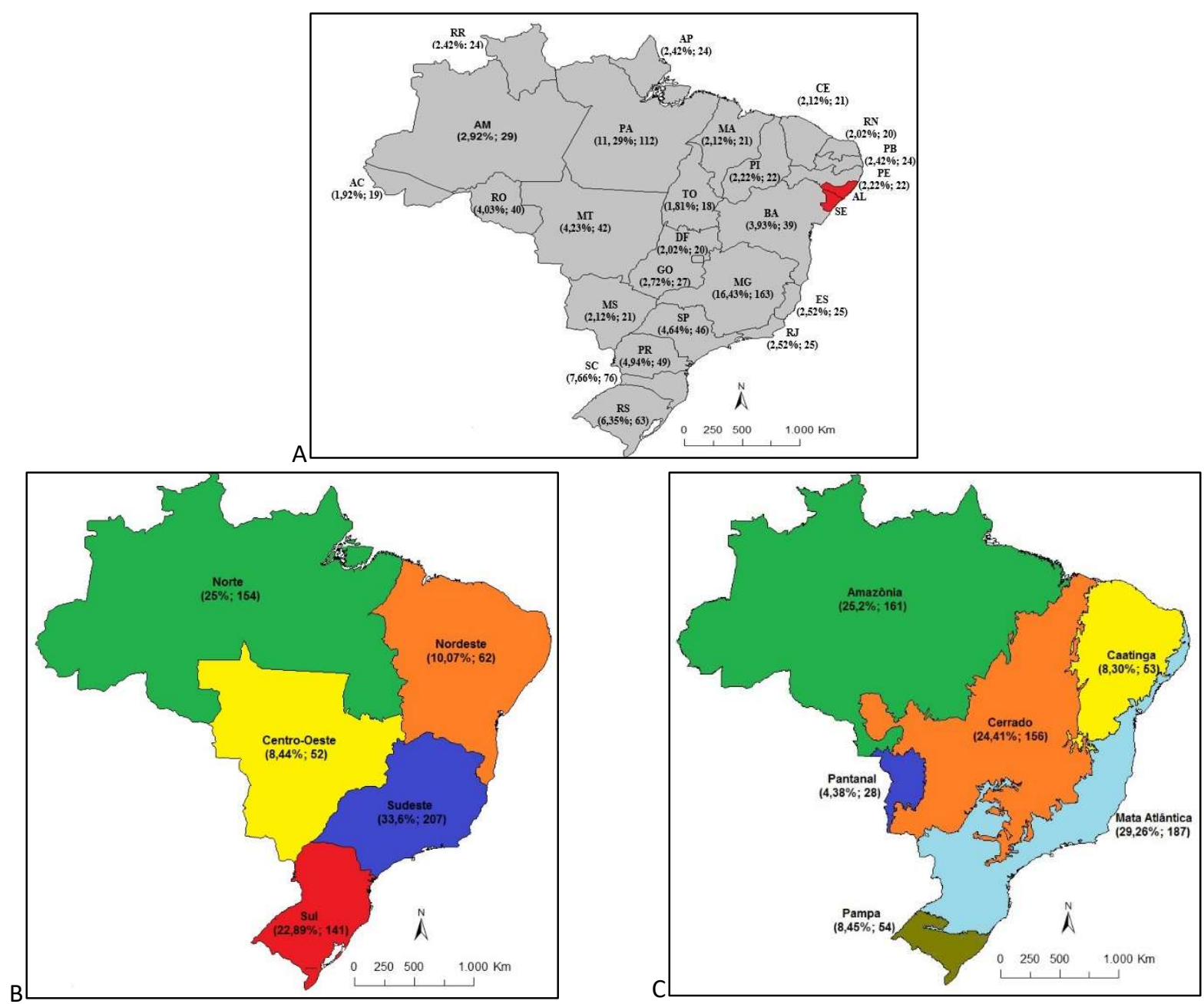

Figura 6: Número e porcentagem de trabalhos sobre mineração publicados nas últimas cinco décadas (1967 - 2017) no Brasil, de acordo com os (A) estados; (B) regiões e (C) biomas brasileiros.

Os estados de Sergipe e Alagoas não apresentaram trabalhos apesar de nesses estados existir atividades minerárias que possuem produtos energéticos como Gás Natural e Petróleo; e minerais não metálicos, como a indústria de água mineral, de agregados minerais, indústria de calcário e cerâmica (BRASIL, 2016).

\section{Minérios estudados nos trabalhos}

Vale ressaltar que durante a pesquisa foram encontrados 80 trabalhos que estudaram mais de um minério, portanto esses trabalhos foram contabilizados nas ocorrências de seus respectivos minérios, por isso o total de 630 minérios estudados. Os minérios com mais frequências nos trabalhos foram o ouro ( $n=165$; $26,19 \%)$, seguido de ferro ( $n=114 ; 18,10 \% ; n=114)$ e carvão mineral ( $n=90 ; 14,29 \%)$, os outros minérios tiveram ocorrências de variação entre 0,32\% ( $n=2)$ a 3,97\% $(n=25)$ (Tabela 1).

A mineração de ouro no país representa $7,04 \%$ da quantidade extraída e exportada para exterior. No ano de 2017 este minério apresentou queda de 76,17\% da produção e no preço (60,75\%), tanto em seu produto primário ou secundário (BRASIL, 2017). Suas principais minas de extração estão localizadas nos 
estados do Pará e Minas Gerais.

Tabela 1: Minérios estudados nos trabalhos publicados sobre mineração no Brasil nas últimas cinco décadas (1967 a 2017).

\begin{tabular}{|c|c|c|}
\hline Minérios & Porcentagem & Quantidade (N) \\
\hline Ouro & $26,19 \%$ & 165 \\
\hline Ferro & $18,10 \%$ & 114 \\
\hline Carvão Mineral & $14,29 \%$ & 90 \\
\hline Bauxita & $4,60 \%$ & 29 \\
\hline Chumbo & $3,97 \%$ & 25 \\
\hline Urânio & $3,65 \%$ & 23 \\
\hline Areia & $2,06 \%$ & 13 \\
\hline Cobre & $2,06 \%$ & 13 \\
\hline Diamantes & $2,06 \%$ & 13 \\
\hline Calcário & $1,90 \%$ & 12 \\
\hline Manganês & $1,59 \%$ & 10 \\
\hline Pedra Britada & $1,59 \%$ & 10 \\
\hline Zinco & $1,59 \%$ & 10 \\
\hline Argila & $1,43 \%$ & 9 \\
\hline Estanho & $1,43 \%$ & 9 \\
\hline Amianto & $0,79 \%$ & 5 \\
\hline Caulim & $0,79 \%$ & 5 \\
\hline Opalas & $0,79 \%$ & 5 \\
\hline Basalto & $0,63 \%$ & 4 \\
\hline Esmeraldas & $0,63 \%$ & 4 \\
\hline Fosfato & $0,63 \%$ & 4 \\
\hline Quartzo & $0,63 \%$ & 4 \\
\hline Xisto & $0,63 \%$ & 4 \\
\hline Ametista & $0,48 \%$ & 3 \\
\hline Arsênio & $0,48 \%$ & 3 \\
\hline Carvão Vegetal & $0,48 \%$ & 3 \\
\hline Gás Natural & $0,48 \%$ & 3 \\
\hline Gipsita & $0,48 \%$ & 3 \\
\hline Petróleo & $0,48 \%$ & 3 \\
\hline Prata & $0,48 \%$ & 3 \\
\hline Talco & $0,48 \%$ & 3 \\
\hline Titânio & $0,48 \%$ & 3 \\
\hline Topázio & $0,48 \%$ & 3 \\
\hline Água Mineral & $0,32 \%$ & 2 \\
\hline Cálcio & $0,32 \%$ & 2 \\
\hline Cromita & $0,32 \%$ & 2 \\
\hline Granito & $0,32 \%$ & 2 \\
\hline Mármore & $0,32 \%$ & 2 \\
\hline Nióbio & $0,32 \%$ & 2 \\
\hline Níquel & $0,32 \%$ & 2 \\
\hline Pirocloro & $0,32 \%$ & 2 \\
\hline Saibro & $0,32 \%$ & 2 \\
\hline Scheelita Verde & $0,32 \%$ & 2 \\
\hline Total & $100,00 \%$ & 630 \\
\hline
\end{tabular}

As produções científicas destinadas a estudar a produção de ouro podem estar relacionadas ao conhecimento das características tecnológicas do minério, que ainda são escassas principalmente quanto a precisão dos dados. A possibilidade de geração de conhecimento através dos estudos das características das associações mineralógicas do ouro pode propiciar melhorias no planejamento de lavras e no desempenho do processamento do minério da usina, o que representa melhor aproveitamento dos recursos minerais e melhor custo (COSTA, 2016). 


\section{Impactos socioambientais estudados nos trabalhos}

A associação dos dois eixos da PCoA representou 74,34\% da variação dos dados. A PCoA 1 explicou sozinha 60,99\% dos nossos resultados e a PCoA 2 representou 13,35\% dos trabalhos. Nessa análise foi observado que houve uma separação dos trabalhos por tipo de ambiente (terrestre e aquático). Existe uma relação do impacto 'alteração da qualidade da água' (ALQAG) aos trabalhos desenvolvidos em ambientes aquáticos, os demais impactos estão relacionados aos estudos desenvolvidos em ambientes terrestres (Figura 7a).

Existe diferença significativa entre os tipos de impacto socioambiental abordados nos trabalhos sobre mineração no Brasil ( $F=2.1043 ; p=0,042$ ) (Figura 7b). As produções científicas analisadas neste estudo demonstram que os impacto associado a extração de minérios mais estudados nos últimos cinquenta anos está relacionado a alteração da qualidade do solo (ALQSO), seguido de alteração da qualidade da água (ALQAG) e alteração da qualidade da saúde humana (ALQSH) (Figura 7b).
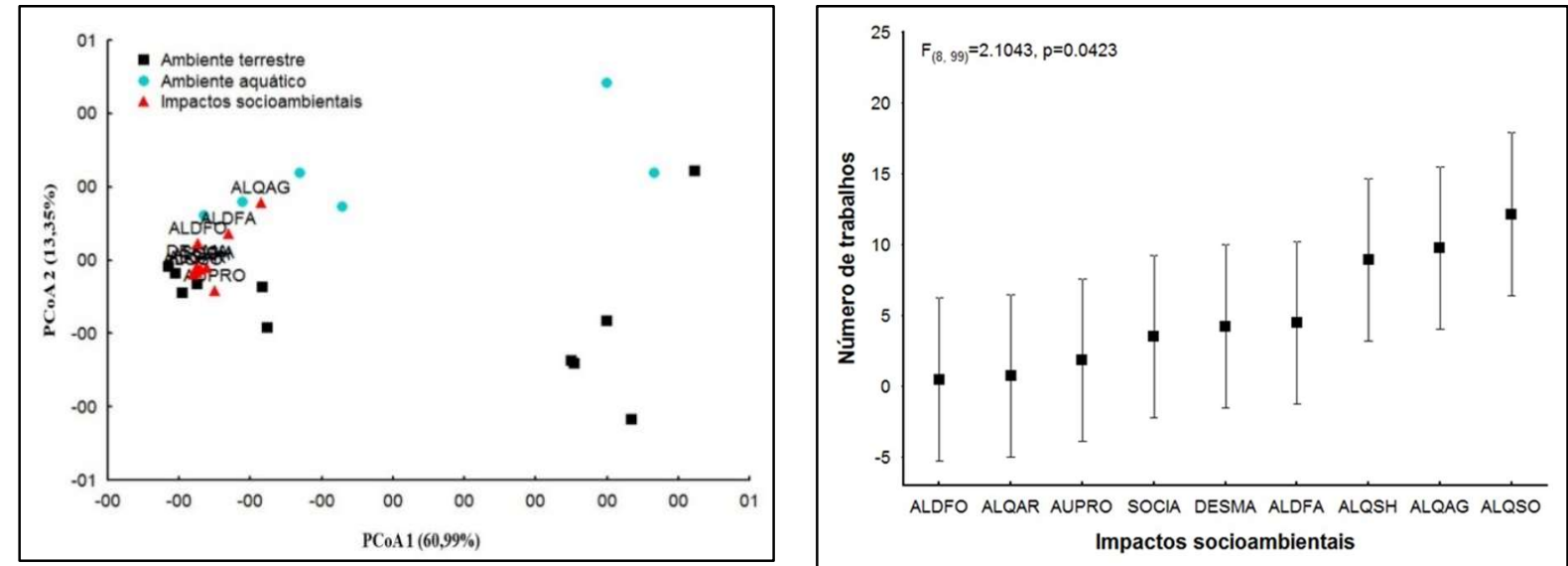

Figura 7: (A) Ordenação gerada pela Análise de Coordenadas Principais (PCoA) para a composição de impactos associados aos ambientes terrestres e aquáticos (B) Comparação do número de trabalhos publicados sobre mineração no Brasil nas últimas cinco décadas (1967 a 2017) por tipo de impacto socioambiental. Legenda: ADFO=Alteração da qualidade da flora; Alteração da qualidade do ar (ALQAR), Aumento da produtividade (AUPRO), Social (SOCIA), Desmatamento (DESMA, Alteração da qualidade da fauna (ADFA), Alteração da qualidade da saúde humana (ALQSH), Alteração da qualidade da água (ALQAG), Alteração da qualidade do solo (ALQSO).

As pesquisas com intuito de estudar impactos da alteração da qualidade do solo tiveram mais dominância devido a mineração ser um fator de degradação do ecossistema, onde a retirada da vegetação irá ocasionar impactos diretos no solo, como aceleração dos processos erosivos ocasionados pela ação direta da água da chuva e do vento, redução da fertilidade do solo, contaminação do solo, assoreamento dos mananciais e perda de mesofauna. Portanto as atividades que envolvem a extração de minérios são consideradas com um potencial de degradação do solo maior e uma recuperação mais difícil, demorada e normalmente de mais alto custo, porém não impossível de ser recuperada como vários projetos implantados em diferentes regiões (MARTINS, 2016).

Outro impacto que obteve destaque associado ao tipo de ambiente (aquático), foi a alteração da qualidade da água, como a contaminação ocasionada por metais pesados, a liberação desses poluentes para o meio pode ocorrer por meio da lixiviação de rejeitos estocados, dos efeitos de erosão e do rompimento de 
barragens e, uma vez nos corpos aquáticos, podem ser transportados ocasionando contaminação de ambientes distantes das fontes de poluição (MELO et al., 2012).

\section{Áreas de atuação dos autores}

A área com maior frequência de ocorrência foi Ciências Biológicas com ( $n=238 ; 43,27 \%)$ e Ciências Exatas e da Terra ( $n=128 ; 23,27 \%$ ) e Ciências da Saúde ( $n=49 ; 8,91 \%)$. Os resultados demonstram que a área de ciências biológicas tem sido a responsável pela porcentagem considerável de autores que estão desenvolvendo trabalhos na temática no decorrer dos últimos cinquenta anos. O profissional da grande área das ciências biológicas tem realizados trabalhos para investigar a contaminação de mananciais próximos a locais de exploração de minérios, contaminação do solo e desenvolvimento de tecnologias para o setor minerário.

O número expressivo de estudos realizados por pesquisadores da área de Ciências Biológicas e pela segunda grande área do conhecimento Ciências Exatas e da Terra, pode ser interligado aos determinantes da temática ser voltada a assuntos de competências científicas das respectivas áreas, maiores números de vagas nas instituições de ensino superior e ao fato que nas últimas décadas o Brasil vem experimentando crescimento expressivo em seus indicadores de desenvolvimento científico. A formação de recursos humanos altamente qualificados, como a formação de doutores, em especial nos últimos anos, assim como o aumento do investimento em ciência, tecnologia e inovação, tem colaborado para que o país esteja entre os 20 países que mais investem em pesquisa e desenvolvimento e consequentemente tenha o aumento na publicação de documentos científicos (CILONI et al., 2013).

\section{CONCLUSÕES}

Avaliar as métricas de produção científica da área de mineração no Brasil, permitiu identificar os estudos dos impactos socioambientais decorrentes da mineração, seja o impacto positivo ou negativo, fator que contribui positivamente para que possíveis novos pesquisadores possam vir a realizar estudos que contemplem essa temática, salientando a necessidade de se descentralizar as pesquisas dos maiores centros urbanos do país e expandi-las as demais localidades.

Quando se leva em consideração o tipo de ambiente em que essa atividade mais está sendo estudada, trabalhos em ambientes terrestres e aquático possuem o maior índice de pesquisas, características mencionadas por autores acerca destes ambientes, ressaltam a necessidade de realizar estudos para possibilitar a recuperação destes ambientes afetados direta ou indiretamente pela atividade de extração mineral, além de novos estudos para desenvolvimento de novas tecnologias mais sustentáveis.

Outro ponto ressaltado pelo estudo se dá acerca das lacunas a serem preenchidas como os estados que a ocorrência de pesquisas sobre essa atividade ainda tem certa carência de estudos, propõe-se um maior investimento com pesquisas voltadas para os estados de Sergipe e Alagoas, ao que permite afirmar que estes estados não apresentaram estudos.

Os resultados alcançados permitiram uma visão abrangente da grande importância da mineração, 
seus benefícios para a economia e malefícios decorrentes de suas atividades para o aspecto socioambiental, no quais os trabalhos encontrados mostraram a indispensabilidade da existência de investigação de novas técnicas e aperfeiçoamento das existentes no processo de recuperação de áreas degradadas. Por essa razão faz-se necessário à realização de novas pesquisas que buscam conciliar a atividade de mineração a um desenvolvimento mais próximo do sustentável possível.

Através da cienciometria, pôde-se notar uma tendência de assuntos estudados nas pesquisas associadas a acontecimentos da temática, as eventualidades ocorridas nas décadas nortearam os rumos das pesquisas, aja visto que a ciência está fortemente influenciada pelas questões socioambientais e políticas que rodeiam, buscando sempre inovações em técnicas sustentáveis que auxiliem o desenvolvimento socioeconômico do país.

AGRADECIMENTOS: ao Instituto de Ciências e Tecnologia das Águas (ICTA) da Universidade Federal do Oeste do Pará (UFOPA) e à Coordenação de Aperfeiçoamento de Pessoal de Nível Superior (CAPES).

\section{REFERÊNCIAS}

AKERMAN, M.. Medidas de experiência e cienciometria para avaliar impacto da produção científica. Revista Saúde Púbica, Santo André, v.47, n.4, p.824-8, 2013. DOI: http://doi.org/10.1590/S0034-8910.2013047004756

ALMEIDA, E. C. E.; GUIMARÃES, J. A.. Brazil's growing production of scientific articles-how are we doing with review articles and other qualitative indicators?. Journal Scientometrics, Rio de Janeiro, v.97, n.2, p.287-315, 2013. DOI: http://doi.org/10.1007/s11192-013-0967-y

ALVES, M. T. R.; TERESA, F. B.; NABOUT, J. C.. A global scientific literature os research on water quality índices: trends, biases and future directions. Acta Limnologica Brasiliensia, Rio Claro, v.26, n.3, p.245-253, 2014. DOI: http://doi.org/10.1590/S2179-975×2014000300004

ALVARADO, R. U.. A bibliometria, informetria, cienciometria e outras "metrias" no brasil. In: ENCONTRO BRASILEIRO DE BIBLIOMETRIA E CIENTOMETRIA, 4. Anais. Recife: EBBC/BRAPCI, 2014.

ARAUJO, E. R.; OLIVERI, R. D.; FERNANDES, F. R. C.. Atividade mineradora gera riqueza e impactos negativos nas comunidades e no meio ambiente. In: Recursos Minerais e Sociedade: Impactos Humanos - Socioambientais Econômicos. Rio de Janeiro: CETEM/MCTI, 2014.

AUMOND, J. J.; MACANAEIRO, J. P.. Abordagem sistêmica e aplicação de rugosidades para desencadear propriedades emergentes em restauração de solos degradados. Revista Arvóre, Santa Maria, v.24, n.3, p.759-764, 2014.

BATISTA, S. G. M.; BARRETO-GARCIA, P. A. B.; SANTOS, L. M.; PAULA, A.. Análise cienciométrica de produções científicas sobre serapilheira no Brasil, Sodebras, v.11, n.128, p.102105, 2016

BARBOSA, F. T.; SOUZA, D. A.. Frequência do uso adequado nos testes estatísticos nos artigos originais publicados na revista brasielira de anestesiologia entre janeiro de 2008 e dezembro de 2009. Revista Brasielira de Anestesiologia, Campinas, v.60, n.5, p.528-536, 2010. Dol: http://doi.org/10.1590/S0034-70942010000500009

BITENCOURT, D. G. B.; PINTO, L. F. S.; PAULETTO, E. A.; SILVA, M. T.; GARCIA, G. F.. Geração de drenagem ácida e de contaminação por metais pesados em perfis de solos construídos em área de mineração de carvão. Revista Brasileira de Ciência do Solo, Viçosa, v.39, p.1821-1834, 2015. DOI: http://doi.org/10.1590/01000683rbcs20140810

BROFMAN, P. R.. A Importância das publicações científicas. Cogitare Enfermagem, v.17, n.3, p.419-21, 2012.

CABRAL-NETTO, O. V.; LAURINDO, F. J. B.. Uma análise cienciométrica da literatura de inteligência competitiva. Production, São Paulo, v.25, n.4, p.764-778, 2015. DOI: http://doi.org/10.1590/0103-6513.063411

CAMPOS, F. S.; BRITO, D.; SOLÉ, M.. Diversity patterns, research trends and mismatches of the investigative efforts to amphibian conservation in Brazil. Anais da Academia Brasileira de Ciências, Rio de Janeiro v.86, n.4, p.1873-1886, 2014. DOI: http://doi.org/10.1590/0001-3765201420140170

CILONI, A. D.; BERBERT, C. O.. As unidades de pesquisa do Ministério de Ciência, Tecnologia e Inovação (MCTI) no contexto da ciência brasileira. Parcerias Estratégicas, Brasília, v.18, n.37, p.83-102, 2013.

CINTRA, P. R.. A produção cientifica sobre docência no ensino superior: uma análise bibliométrica da Scielo Brasil. Avaliação: Revista da Avaliação da Educação Superior, Campinas, v.23, n.2, p.567-585, 2018. DOI: http://doi.org/10.1590/S1414-40772018000200016

COSTA, F. R.. Caracterização tecnológica do minério de ouro da Mina Morro do Ouro-Paracatu-MG. Dissertação 
(Mestrado em Ciências) - Universidade de São Paulo, São Paulo, 2016.

CHIARINI, T.; OLIVEIRA, V. P; SILVA NETO, F. C. C.. Spatial distribution of scientific activities: An exploratory analysis of Brazil, 2000-10. Science and Public Policy, v.41, n.5, p.625640, 2014. DOI: http://doi.org/10.1093/scipol/sct093

DORNELES, A. B.. Análise da Produção Acadêmica no Campo da Educação Ambiental: um Olhar Cienciométrico (19922016). Monografia (Graduação em Engenharia Florestal) Universidade de Brasília, Brasília, 2016.

BRASIL. Departamento Nacional de Produção Mineral. Anuário Mineral Brasileiro: Principais Substâncias Metálicas, Brasília: DNPM, 2016.

BRASIL. Deparatamento Nacional de Produção Mineral. Informe mineral 20 semestre/2017. Brasília: DNPM, 2017.

FANTIN-CRUZ, I.; CAMARGO, J. C.. Estudo cienciométrico sobre a docência no ensino superior no Brasil. Espacios, v.36, n.6, p.3, 2015.

FIGUEIREDO-FILHO, D. B.; SILVA JÚNIOR, J. A.. Desvendando os Mistérios do Coeficiente de Correlação de Pearson ( $r$ ). Revista Politica Hoje, v.18, n.1, 2009.

LAURINDO, R.; MAFRA, T.. Cienciometria da revista Comunicação \& Sociedade identifica interfaces da área. Comunicação \& Sociedade, São Bernardo do Campo, v.53, n.31, p.233-260, 2010. DOI: http://doi.org/10.15603/21757755/cs.v31n53p233-260

LEGENDRE, P.; LEGENDRE, L.. Numerical ecology. 2 ed. Amsterdam: Elsevier, 1998.

LINS, F. A. F.. Tecnologia mineral: pesquisa, desenvolvimento e inovação. In: MELFI, A. J.; MISI, A.; CAMPOS, D. A.; CORDANI, U. G.. Recursos Minerais no Brasil: Problemas e Desafios. Rio de Janeiro: Academia Brasileira de Ciências, 2016. p.244-247

MACIAS-CHAPULA, C. A.. O papel da informetria e da cienciometria e sua perspectiva nacional e internacional. Ciência da Informação, Brasília, v.27, n.2, p.134-140, 1998. DOI: http://doi.org/10.18225/ci.inf.v27i2.794

MARTINS, S. V.. Recuperação de áreas degradadas: ações em áreas de preservação permanente, voçorocas, taludes rodoviários e de mineração. Viçosa: Aprenda Fácil, 2016.

MARÔCO, J.. Análise Estatística com o SPSS Statistic. Lisboa: ReportNumber, 2018.

MELLO, R. C. A.. Análise do aporte de sedimentos na bacia hidrográfica do córrego Assistência, SP: caso do Polo Cerâmico de Santa Gertrudes. Dissertação (Mestrado em Geociências e Meio Ambiente) - Universidade Estadual Paulista "Júlio de Mesquita Filho", Rio Claro, 2015.

MELO, V. F.; ANDRADE, M.; BATISTA, A. H.; FAVARETTO, N.. Chumbo e zinco em águas e sedimentos de área de mineração e metalurgia de metais. Química Nova, São Paulo, v.35, n.1, p.22-29, 2012. DOI: http://doi.org/10.1590/S0100-40422012000100005
MOTA, J. A.; SOUZA-FILHO, P. W. M.; TORRES, V. F. N.; SIQUEIRA, J. O.; SANTOS, J. F.; MATLABA, V.. Uma nova proposta de indicadores de sustentabilidade na mineração. Sustentabilidade em Debate, Brasília, v.8, n.2, p.15-29, 2017. DOI: http://doi.org/10.18472/SustDeb.v8n2.2017

MUGNAINI, R.; JANNUZZI, P. M.; QUONIAM, L.. Indicadores bibliométricos da produção científica brasileira: uma análise a partir da base Pascal. Ciência da Informação, Brasília v.33, n.2, p.123-131, 2004.

NICACIO, G.; JUEN, L.. Chironomids as indicators in freshwater ecosystems: na assessment of the literature. Insect Conservation and Diversity, v.8, p.393-403, 2015. DOI: http://doi.org/10.1111/icad.12123

OMACHI, G. Y.. Estudos para o aumento da vida útil das minas de minério de ferro do Quadrilátero Ferrífero, MG. Dissertação (Mestrado em Engenharia Mineral) Universidade Federal de Ouro Preto, Ouro Preto, 2015.

PASSOS, F. L.; COELHO, P.; DIAS, A.. (Des) territórios da mineração: planejamento territorial a partir do rompimento em Mariana, MG. Caderno Metropóles, São Paulo, v.19, n.38, p.269-297, 2017. DOI: http://doi.org/10.1590/22369996.2017-3811

PEREIRA, I. R.; D'ABADIA, P. L.; PRADO, A. D. L.; MATOS, F. S.; NABOUT, J. C.; GONÇALVES, P. J.; ALMEIDA, L. M.. Trends and gaps in the global scientific literature about Jatropha curcas L. (Euphorbiaceae), a tropical plant of economic importance. Semina. Ciências Agrárias, Recife, v.39, n.1, p.718, 2018. DOI: http://doi.org/10.5433/16790359.2018v39n1p7

PUCCINI, L. R. S.; GIFFONI, M. G.; SILVA, L. F.; UTAGAWA, C. Y.. Comparativo entre as bases de dados PubMed, SciELO e Google Acadêmico com o foco na temática 53 Educação Médica. Cadernos UnifOA, Volta Redonda, v.28, p.75-82, 2015.

RAZERA, J. C. C.. Contribuições da cienciometria para a área brasileira de Educação em Ciências. Ciência \& Educação, São Paulo, v.22, p.3, p.557-560, 2016. DOI http://doi.org/10.1590/1516-731320160030001

ROCHA, E. M.; MORAES, L. G. M.; ALMEIDA, L.V.; DALVI, L. R.; ANDRIATO, L. C.; BERGAMASCHI, L. K.; BERNARDINA, L. S. D.; PEREIRA, W. B.; GIMENEZ, V. G.; CHIARELLI-NETO, O.; ALMEIDA, H. S.. Impacto do rompimento da barragem em Mariana-MG na saúde da população ribeirinha da cidade de Colatina-ES. Tempus Actas de Saúde Coletiva, Brasília, v.10, n.3, p.31-45, 2016. DOI: http://doi.org/10.18569/tempus.v10i3.1902

REIS, D. A.; LIMA, C. L. R .; PAULETTO, E. A.. Resistência tênsil de agregados e compressibilidade de um solo construído com plantas de cobertura em área de mineração de carvão em Candiota, RS. Revista Brasileira de Ciência do Solo, Viçosa, v.38, n.2, p.669-678, 2014. DOI: http://doi.org/10.1590/S0100-068320014000200031

SANTOS, N. M.; ACCIOLY, A. M. A.; NASCIMENTO, C. W. A.; SILVA, I. R.; SANTOS, J. A G.. Biodisponibilidade de chumbo por extratores químicos em solo tratado com ácidos húmicos e carvão ativado. Revista Ciência Agronômica, Fortaleza, 
v.46, n.4, p.663-668, 2015. DOI:

http://doi.org/10.5935/1806-6690.20150052

SIDONE, O. J. G.; HADDAD, E. A.; MENA-CHALCO, J. P.. A Ciência nas Regiões Brasileiras: Evolução da Produção e das Redes de Colaboração Científica. Transiformação, Campinas, v.28, n.1, p.15-31, 2016. DOI: http://doi.org/10.1590/231808892016002800002

SPINAK, E.. Dicionario enciclopédico de bibliometría, cienciometría e informetría. Caracas: UNESCO, 1996.

TEIXEIRA, A. F. S.; KEMMELMEIER, K.; MARASCALCHI, M. N.; STÜRMER, S. L.; CARNEIRO, M. A. C.; MOREIRA, F. M. S.. Arbuscular mycorrhizal fungal communities in an iron mining area and its surroundings: Inoculum potential, density, and diversity of spores related to soil properties. Ciência e Agrotecnologia, Lavras, v.41, n.5, p.511-525, 2017. DOI: http://doi.org/10.1590/1806-90882017000600010

VANTI, N. A.. Da bibliometria à webometria: uma exploração conceitual dos mecanismos utilizados para medir o registro da informação e a difusão do conhecimento. Ciência da Informação, Brasília, v.31, n.2, p.152-162, 2002. DOI: http://doi.org/10.1590/S0100-19652002000200016

VIEIRA, E. G.; REZENDE, E. N.. Exploração de areia em um ambiente ecologicamente equilibrado: é possível conciliar? Sustentabilidade em Debate, Brasília, v.6, n.2, p.171-1992, 2015. DOI:

http://doi.org/10.18472/SustDeb.v6n2.2015.10795

XING, Y.; SI, Y. X.; HONG, C.; LI, Y.. Multiple factors affect diversity and abundance of ammonia-oxidizing microorganisms in iron mine soil. Archives of Environmental Contamination and Toxicology, v.69, n.1, p.20-31, 2015. DOI: http://doi.org/10.1007/s00244-015-0144-9

WEBER, C. C.. A mineração Mineral de carvão mineral no Rio Grande do Sul e a recuperação ambiental das áreas degradadas. Monografia (Especialização em Direito Ambiental) - Universidade Federal do Rio Grande do Sul, Porto Alegre, 2014.

A CBPC - Companhia Brasileira de Produção Científica (CNPJ: 11.221.422/0001-03) detém os direitos materiais desta publicação. Os direitos referem-se à publicação do trabalho em qualquer parte do mundo, incluindo os direitos às renovaç̃oses, expansões e disseminações da contribuiç̃o, bem como outros direitos subsidiários. Todos os trabalhos publicados eletronicamente poderão posteriormente ser publicados em coletâneas impressas sob coordenação da Sustenere Publishing, da Companhia Brasileira de Produção Científica e seus parceiros autorizados. Os (as) autores (as) preservam os direitos autorais, mas não têm permissão para a publicação da contribuição em outro meio, impresso ou digital, em português ou em tradução. 